At 11:50 PM, the emergency management headquarters of prefectural medical response was established in the hospital, but a connection could not be established to 10 regional health centers for the weekend. Water levels of some rivers were increasing nearly to flood levels, and an evacuation order was announced to hundreds of thousands of people. This situation continued for a few days throughout many regions. The information of flood or landslide probability was continuously monitored, but an attempt was made to decide the timing of cancellations of standby.

Discussion: An ordinary response to disaster depends on a clear turning point, such as the occurrence time. In heavy rainfall, there are two issues. One is about actions to prevent disaster and another is a recognition of geographic points or surface. Many critiques to the response focus on the judgments and actions for prevention before a critical event. Lessons learned included the importance of preventive actions along with a timeline and the judgment of restoration.

Prehosp. Disaster Med. 2019;34(Suppl. 1):s43-s44

doi:10.1017/S1049023X19001055

The Role of Japan DMAT in Tokyo Inland Earthquake Dr. Yuichi Koido ${ }^{1}$, Dr. Mibo Misaki ${ }^{1}$, Ms. Kayako Chishima ${ }^{1}$, Dr. Yuzuru Kawashima ${ }^{1}$, Dr. Hisayoshi Kondo ${ }^{1}$,

Mr. Yoshiki Toyokuni ${ }^{1}$, Dr. Yasubiro Otomo ${ }^{2}$,

Dr. Katsunori Yoshihara ${ }^{3}$

1. National Disaster Medical Center of Japan, Tachikawa, Japan

2. Tokyo Medical and Dental University, Bunkyo-ku, Japan

3. Toho University, Ota-ku, Japan

Introduction: An inland earthquake is expected to occur in Tokyo in the near future, and disaster preparedness and response measures have been put in place by the government of Japan and local authorities.

Methods: Japan Disaster Medical Assistant Teams (DMATs) conducted two large-scale drills for the first time in preparation for a Tokyo inland earthquake, in collaboration with the following participants: the Tokyo Metropolitan Government, disaster base hospitals in Tokyo, three Staging Care Units (SCUs), and neighboring prefectures. One of the scenarios was a north Tokyo Bay earthquake affecting the Tokyo wards and had 142 Japan DMATs participation. Another scenario was Tama inland earthquake affected mid-west of Tokyo and 110 DMATs participated. The drill included headquarters operation, affected hospital support operation, patient transportation within the area and to the wider region, SCU operation, collaboration with associated organizations, and logistics operation.

Results: Post-drill assessments identified the following areas that need to be addressed: review of Japan DMAT implementation strategies; improvement of SCUs; establishment of a patient air transportation framework; securing means of patient transportation; improvement of communication systems; strengthening of disaster response of all hospitals in the Tokyo Metropolis; and preparations for survival in the event of isolation caused by the disaster.

Prehosp. Disaster Med. 2019;34(Suppl. 1):s44

doi:10.1017/S1049023X19001067

\section{Study of Medical Supply and Demand Balance for the} Nankai Trough Earthquake

Mr. Yosuke Takada ${ }^{1,2}$, Dr. Yasubiro Otomo ${ }^{1}$

1. Graduate School of Medical and Dental Sciences, Tokyo Medical and Dental University, Tokyo, Japan

2. Dentistry and Pharmaceutical Sciences, Okayama University Graduate School of Medical, Okayama, Japan

Introduction: The Nankai Trough, marking the boundary between the Eurasian Plate and the Philippine Sea Plate, is forecasted to create a tragic earthquake and tsunami within 30 years.

Aim: To clarify the gap between medical supplies and demand. Methods: Collected the data of the estimation of injured persons from each prefecture throughout Japan, and also the number of Intensive Care Unit (ICU) and High Care Unit (HCU) beds in Japan from the Ministry of Health database. We re-calculated the number of severe cases based on official data. Moreover, we calculated the number of beds of hospitals with the capacity to receive severe patients.

Results: The total number of disaster base hospitals is 723 hospitals with 6556 ICU beds, and 545 hospitals have 5,248 HCU beds throughout Japan. When the Nankai Trough earthquake occurs, 187 disaster base hospitals would be located in the area with seismic intensity 6-upper on the Japanese Seismic Intensity Scale of 0-7, and 79 disaster base hospitals would be located in the tsunami inundation area. The estimated total number of injured persons is 661,604 including 26,857 severe cases, 290,065 moderate cases, and 344,682 minor cases.

Discussion: Even if all ICU and HCU beds are usable for severe patients, there will be 15,053 more beds needed. The Cabinet Office of Japan assumes that $60 \%$ of hospital beds would not be able to be used in an area of the seismic intensity of 6-upper. If $80 \%$ of beds are used in the non-disaster time, the number of beds which are usable at the time of a disaster will decrease more. The beds needed for severe patients would be significantly lacking when the Nankai Trough earthquake occurs. It will be necessary to start treatment of the severe patients who are "more likely to be saved more."

Prehosp. Disaster Med. 2019;34(Suppl. 1):s44

doi:10.1017/S1049023X19001079

\section{They have Arrived! How Dallas, Texas Provided Shelter-Based Onsite Medical Care to Evacuees from Hurricane Harvey \\ Dr. Lindsay A. Flax, Dr. E. Liang Liu, Dr. Kelly R. Klein, Dr. Raymond L. Fowler, Dr. Raymond E. Swienton \\ Department of Emergency Medicine, University of Texas \\ Southwestern Medical Center, Dallas, United States}

Introduction: After Hurricane Harvey and the flooding that ensued, 3,829 displaced persons were transported from their homes and sheltered in the Dallas Convention Center. This large general population sheltering operation was medically supported by the onsite Mega-Shelter Medical Clinic (MMC). In an altered standard of care environment, a number of multi-disciplinary medical services were provided including emergent management, acute pediatric and adult care, 
psychiatric/behavioral services, onsite pharmaceutical, and durable medical equipment distribution, epidemiologic surveillance, and select laboratory services.

Aim: To describe how onsite medical care in the adapted environment of a large population shelter can provide comparable services and limit the direct impact on the local medical community.

Methods: A retrospective chart review of medical records was generated for all clinical encounters at the MMC. Data were sorted by daily census, disease surveillance, medical decision making, treatment, and transport destinations.

Results: $40.7 \%$ of registered evacuees utilized the MMC accounting for a total of 2,654 clinic visits by 1,560 unique patients representing all age groups. During the sustained MMC operations, $8 \%$ of patients required emergency transport and 500 additional patient transports were arranged for clinic appointments. No deaths occurred and no iatrogenic morbidity was reported.

Discussion: Medical care was provided for a large number of evacuees which mitigated the potential impact on the local medical infrastructure. The provision of medical services in a large population shelter may necessitate adaptation to the standard of care. However, despite the nontraditional clinical setting, care delivery was not compromised.

Prehosp. Disaster Med. 2019;34(Suppl. 1):s44-s45

doi:10.1017/S1049023X19001080

Typhoon Mangkhut Case Study: Household and Community Typhoon Preparedness in Hong Kong, a Densely Populated Urban City

Ms. Asta YT Man ${ }^{1,2}$, Prof. Emily Ying Yang Chan ${ }^{1,2}$,

Dr. Holly CY Lam ${ }^{1,2}$

1. Collaborating Centre for Oxford University and CUHK for Disaster and Medical Humanitarian Response (CCOUC), JC School of Public Health and Primary Care, The Chinese University of Hong Kong, Hong Kong, China

2. Division of Global Health and Humanitarian Medicine, JC School of Public Health and Primary Care, The Chinese University of Hong Kong, Hong Kong, China

Introduction: As a subtropical urbanized city in Southeast Asia, Hong Kong is prone to frequent typhoons. With an increasing number of severe typhoons, usual preparedness measures should be explored to assess their adequacy to safeguard health and wellbeing. Typhoon Mangkhut (2018) serves as an example of the successes and limitations of community preparedness for a severe typhoon.

Aim: To explore how Hong Kong residents prepared for Typhoon Mangkhut and whether their usual preparedness measures provided enough protection.

Methods: A population-based randomized telephone survey of Hong Kong residents $(n=521)$ was conducted soon after Typhoon Mangkhut's landing. Only residents aged 18 or above and understood Cantonese were included. Socio-demographic factors, types of typhoon and general preparedness, risk perception, and impacts from the typhoon were asked. Descriptive characteristics and univariate analysis were used to describe the patterns and associations.

Results: $8.6 \%$ of respondents felt their home was at high risk of danger during typhoons although $33.4 \%$ reported some form of impact from Mangkhut. Over 70\% reported doing at least one typhoon specific preparedness measure. Among those who practiced at least one typhoon specific preparedness measure, $37.2 \%(\mathrm{p}=0.002)$ were affected by the typhoon.

Discussion: Despite the high adaptation of preparedness measures, warranted by the frequent typhoons, Hong Kong residents were not adequately prepared for a severe typhoon. While the early warning system and evacuation of flood-prone areas mitigated some of the impact, unexpected effects such as flying air conditioners, roadblocks affecting employment, swaying buildings, and loss of power supply were not accounted for. Future preparedness for natural disasters which will become more extreme due to climate change and needs to account for unforeseen risks.

Prehosp. Disaster Med. 2019;34(Suppl. 1):s45

doi:10.1017/S1049023X19001092

\section{Typhoon Preparedness Measures of the Hong Kong Public} for Typhoon Mangkhut

Miss. Tiffany Yeung, Ms. Eva CC Lam

Hong Kong Jockey Club Disaster Preparedness and Response

Institute, Hong Kong

Introduction: Climate change has brought more extreme weather events to Hong Kong. The increasing number of powerful tropical cyclones that hammered Hong Kong in recent years reminded the territory to review typhoon preparedness of the community.

Aim: Typhoon Mangkhut slammed Hong Kong in September 2018 and caused significant devastation. Three weeks after the devastation, the Hong Kong Jockey Club Disaster Preparedness and Response Institute commissioned the Public Opinion Programme at The University of Hong Kong to conduct a survey to understand the general Hong Kong public's typhoon preparedness measures and their information seeking behaviors. Methods: A cross-sectional population-based anonymous telephone survey was conducted on a random sample of 1,018 Cantonese-speaking adults aged 18 or above.

Results: The most common typhoon preparedness measures were taping windows (45\%), followed by food stockpiling (18\%), and closing of doors/windows (10\%). Only $2 \%$ and $1 \%$ of the respondents were prepared for water and power outage, respectively. $36 \%$ of the respondents did not take any precautionary measures. $29 \%$ sought typhoon precautionary measures information from the mass media and 31\% of respondents relied on their previous experience. Other sources of information included government sources (7\%) and social media (7\%).

Discussion: Though no death cases were reported related to Typhoon Mangkhut, the effect of the superstorm caused over 300 casualties, blocked roads, and transportation chaos caused 